\title{
Origami and Partial Differential Equations
}

\section{Bernard Dacorogna, Paolo Marcellini, and Emanuele Paolini}

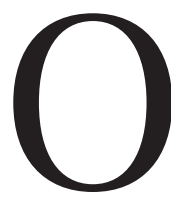

rigami is the ancient Japanese art of folding paper. Even if origami is mainly an artistic product, it has received a great deal of attention from mathematicians, because of its interesting algebraic and geometrical properties.

We present a new mathematical model of origami with a double purpose. On one hand we give an analytical approach which provides a new perspective to the existing algebraic and geometrical models. On the other hand we use origami as a tool to exhibit explicit solutions to some systems of partial differential equations.

Mechanical properties of paper, as a material, are simple. A sheet of paper is rigid in tangential directions. Indeed, it cannot be stretched, compressed, or sheared. If a sheet of paper is constrained on a plane, it would only be possible to achieve rigid motions, i.e., rotations and translations of the whole sheet. On the other hand, in the normal direction it can be easily folded. This is due to the infinitesimal thickness of the paper and to the elastic properties of the fibers composing the material.

The physical properties we have described so far can be defined from a mathematical point of view. To this aim we let $\Omega \subset \mathbb{R}^{2}$ be a two-dimensional domain (usually a rectangle), which is the reference

Bernard Dacorogna is professor of mathematics at the École Polytechnique Fédérale de Lausanne, Switzerland. His email address is bernard. dacorogna@epf1. ch.

Paolo Marcellini is professor of mathematics at the University of Firenze, Italy. His email address is marce11 ini @ math.unifi.it.

Emanuele Paolini is assistant professor of mathematics at the University of Firenze, Italy. His email address is paolini@math.unifi.it.

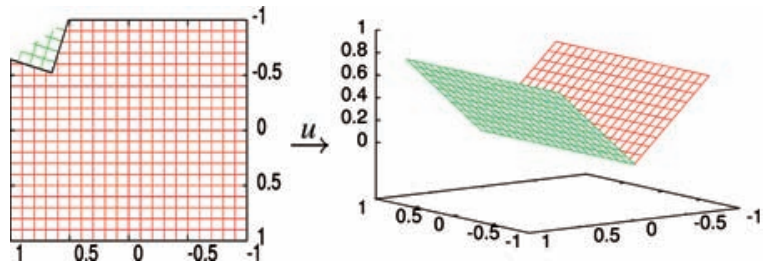

Figure 1. The map $u(x, y)=\left(x, \frac{y}{\sqrt{2}}, \frac{|y|}{\sqrt{2}}\right)$ describes a 90-degree folding in $\mathbb{R}^{3}$ of the square paper $\Omega$ in the left-hand side. The gradient of $u$ is a $3 \times 2$ orthogonal matrix.

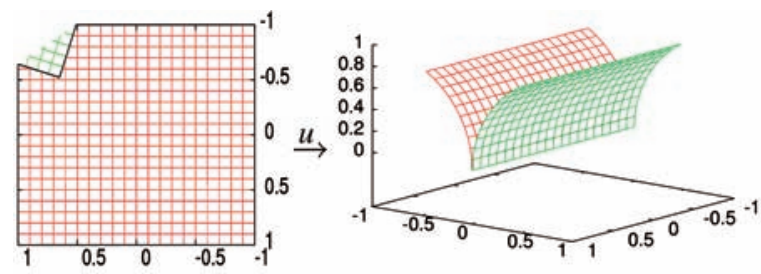

Figure 2. The map $u(x, y)=(x, \operatorname{sign}(y)(1-\cos y), \sin |y|)$, is not piecewise linear. However, the gradient is a $3 \times 2$ orthogonal matrix.

configuration for the sheet of paper. An origami is a suitable immersion of the sheet of paper in the three-dimensional space. Hence it can be identified with a map $u$ (see Figures 1 and 2)

$$
u: \Omega \subset \mathbb{R}^{2} \rightarrow \mathbb{R}^{3} .
$$

The tangential rigidity can be expressed by requiring that $u$ is a rigid map of $\Omega$ into $\mathbb{R}^{3}$. In other words the gradient $D u(x)$ of the map $u$ is an orthogonal $3 \times 2$ matrix, meaning that, 

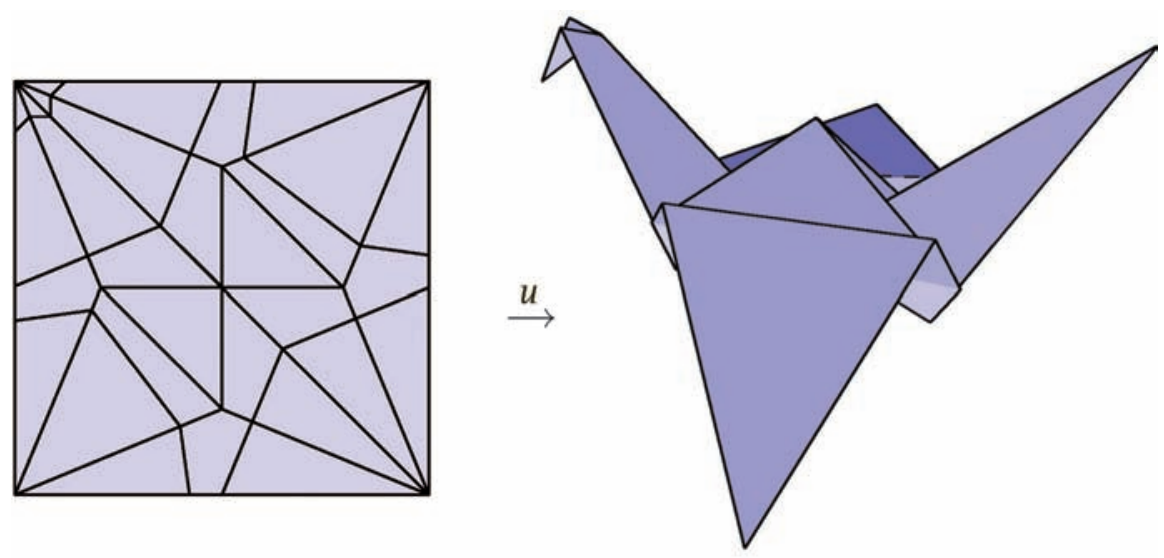

Figure 3. On the right: the crane is the most famous origami; on the left: the corresponding singular set.

for every $x \in \Omega$,

$$
D u(x) \in O(3,2):=\left\{M \in \mathbb{R}^{3 \times 2}: M^{t} M=I\right\} .
$$

Since $D u^{t} D u=I$, the rank of $D u$ is maximal; hence the immersion is locally one-to-one. Thus, if $u$ is sufficiently regular, it is a local isometry between $\mathbb{R}^{2}$ and the manifold $u(\Omega) \subset \mathbb{R}^{3}$ with the metric induced by the ambient space $\mathbb{R}^{3}$. Therefore the Gauss curvature of $u(\Omega)$ coincides with the Gauss curvature of $\Omega$, which is zero, and $u(\Omega)$ is a developable surface (see [15]).

Since origami is a folded paper, the map $u$ cannot be everywhere smooth; it is only piecewise smooth. Folding creates discontinuities in the gradient. Since we do not allow cutting the sheet of paper, $u$ is, however, a continuous map. The singular set $\Sigma_{u} \subset \Omega$, which is the set of discontinuities of the gradient $D u$, is called crease pattern in the origami context. Usually this set is composed by straight segments (as in Figure 3), but it is also possible to make origami with curved folds (see Figure 4).

This formulation of an origami as a map $u: \Omega \subset \mathbb{R}^{2} \rightarrow \mathbb{R}^{3}$ also allows us to consider modular origami, which corresponds to a domain $\Omega$ which is not connected (several sheets of paper), as for instance in Figure 5.

A special attention will be given to the so-called flat origami. A flat origami is defined as a map whose image is contained in a plane; it can be represented, up to a change of coordinates, as a map

$$
u: \Omega \subset \mathbb{R}^{2} \rightarrow \mathbb{R}^{2} .
$$

In this case it can be proved that the folding lines must be straight segments which satisfy some angle condition at every vertex of the singular set $\Sigma_{u}$. More precisely, at any vertex of the singular set $\Sigma_{u}$, exactly $2 k$ consecutive angles $\alpha_{1}, \cdots, \alpha_{2 k}$ meet with the property that

$\alpha_{1}+\alpha_{3}+\cdots+\alpha_{2 k-1}=\alpha_{2}+\alpha_{4}+\cdots+\alpha_{2 k}=\pi$.
We will see (cf. Theorem 4.1) that such condition is enough to guarantee, on a simply connected open set $\Omega$, that a given union of segments $\Sigma$ is actually the singular set $\Sigma_{u}$ of some rigid map $u$.

Our general definition allows us, in a natural way, to consider in the higher-dimensional case a map $u: \Omega \subset \mathbb{R}^{n} \rightarrow \mathbb{R}^{m}$, where the gradient $D u(x)$ is an orthogonal $m \times n$ matrix. That is, $D u(x) \in O(m, n)$, where

$$
O(m, n):=\left\{M \in \mathbb{R}^{m \times n}: M^{t} M=I\right\} .
$$

When $m=n$ we simply write $O(n)$. In this case our mathematical global approach also allows us to consider flat origami in higher dimensions $\left(u: \Omega \subset \mathbb{R}^{n} \rightarrow \mathbb{R}^{n}, D u(x) \in O(n)\right.$ ), which will also be called hyper-origami. As an example, for $n=3$, we will describe, in the section "Fractal Construction", how to "fold" a cube.

Closely related is the more difficult problem of finding a map with orthogonal gradient satisfying some boundary condition. For example, when $n=m=2$, we consider the Dirichlet problem

$$
\left\{\begin{array}{cl}
D u(x) \in O(2) & \text { for a.e. } x \in \Omega \\
u(x)=0 & \text { for } x \in \partial \Omega .
\end{array}\right.
$$

At first sight one could think that, by means of repeated folding, a sheet of paper could in principle be crunched to a single point, say the origin. To convince oneself that this is not possible it is enough to look at the equation. An origami folded to a single point $y_{0}(=0)$ is in fact represented by the constant map $u \equiv y_{0}$, which, however, has gradient $D u \equiv 0 \notin O(2)$.

In order to solve (1), we have to take advantage of the fact that the boundary does not have full dimension. Anyway, the construction cannot be that simple; in fact, it is impossible to find a map solving the system and which is smooth on some neighborhood of a point of the boundary. Indeed, 

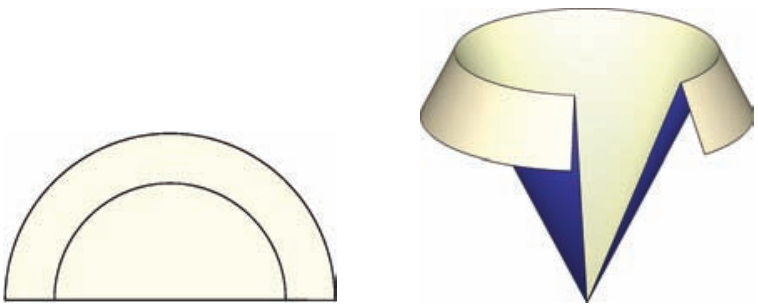

Figure 4. A circular crease can be folded to obtain a cone.

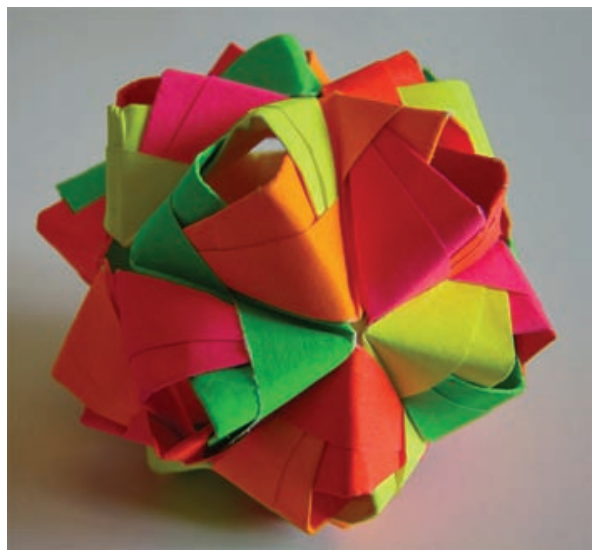

Figure 5. A picture of a modular origami representing an icosahedron, built with 30 identical copies of a folded square, linked together in a symmetrical way.

let $u$ be a smooth map which satisfies $D u \in O(2)$ or equivalently

$$
|D u|^{2}=2|\operatorname{det} D u|=2 .
$$

Since det $D u= \pm 1$, by continuity the Jacobian has a sign, say $\operatorname{det} D u=1$. Therefore the equation $|D u|^{2}=2 \operatorname{det} D u$, with the notation $u(x, y)=$ $\left(\begin{array}{l}v(x, y) \\ w(x, y)\end{array}\right)$, can be easily transformed into the CauchyRiemann system

$$
\left\{\begin{array}{l}
v_{x}-w_{y}=0 \\
v_{y}+w_{x}=0
\end{array}\right.
$$

with furthermore $|D v|=|D w|=1$. Denoting by $\tau, \nu$, respectively, the tangent and normal unit vectors on $\partial \Omega$, we thus have, up to sign,

$$
\langle D v ; \tau\rangle=\langle D w ; v\rangle, \quad\langle D w ; \tau\rangle=\langle D v ; v\rangle .
$$

Since $v=w=0$ on $\partial \Omega$, we also obtain $D v=D w=$ 0 , which contradicts the fact that $|D v|=|D w|=1$.

Thus any solution to the differential problem (1) is Lipschitz continuous but not of class $C^{1}$ near the boundary; therefore it assumes in a fractal way the homogenous boundary datum $\varphi=0$ (see Figure 6). The map $u$ will be explicitly defined at every $(x, y) \in \Omega$, and it will be piecewise affine, with infinitely many pieces, in accord with its fractal nature near the boundary of $\Omega$.
Using this property of the singular set we are able to construct some examples of fractal origami which satisfy some "nonisometric" boundary conditions. In general, solutions to (1) may be very irregular, up to the point that the singular set can be everywhere dense.

In conclusion, origami is seen here as a tool for finding simple solutions in a context of systems of partial differential equations where infinitely many solutions exist, none of them being of class $C^{1}$, most of them having very irregular gradient. General existence theories have been developed by means of convex integration and by Baire category method to obtain Lipschitz continuous solutions. Simpler scalar problems can be treated with the viscosity theory, obtaining explicit representation formulas. This theory cannot, however, be applied in the context considered here, due to the lack of a maximum principle. Nevertheless, origami allows us to present particularly simple solutions with a recursive structure. It is a challenging issue to adapt the method proposed here to more general systems of first-order differential systems such as those encountered in geometry, optimal design, and nonlinear elasticity (see the section: "Implicit Partial Differential Equations: Conclusion”).
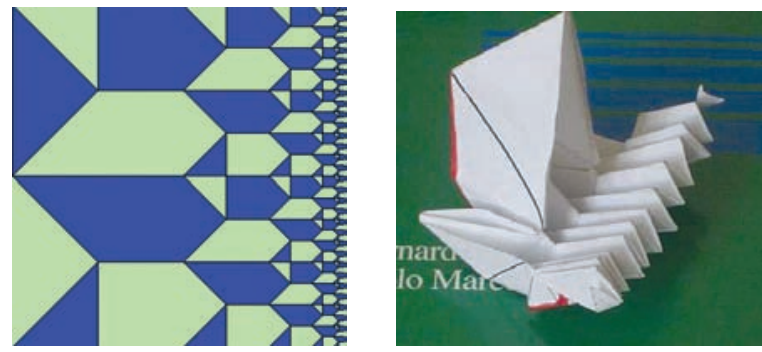

Figure 6. On the right-hand side a solution to the Dirichlet problem (1) actually folded with paper; it gives rise to a fractal shape. In the left-hand side the singular set of the same map is represented in a region near the boundary.

\section{Some Other Topics About Origami}

Many mathematicians interested in geometry or algebra (for example, in group theory, Galois theory, graph theory...) have studied origami constructions. An important issue is the geometrical construction of numbers. In this aspect origami turns out to be more powerful than the classical rule and compass constructions (see [1], [5], [6] or [17]). In order to determine what can be constructed through origami, it is important to formalize the rules. These are known as Huzita axioms and have been proposed by Hatori, Huzita, Justin, and Lang. Here are the seven axioms.

Axiom 1: Given two points $P_{1}$ and $P_{2}$, there is a unique fold passing through both of them. 

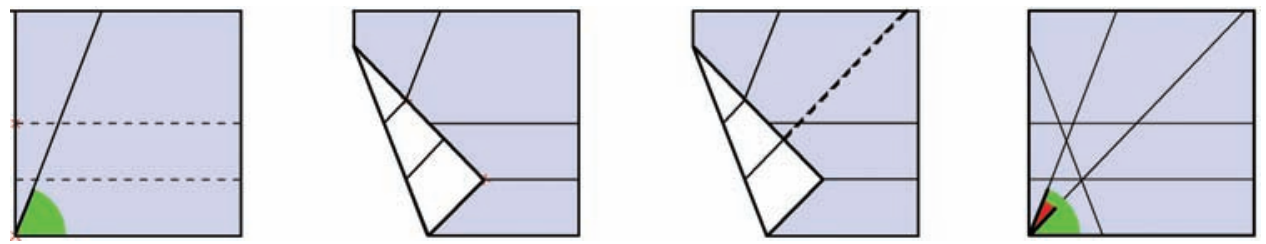

Figure 7. Trisecting an angle. The resulting red angle is a third of the given green angle.

Axiom 2: Given two points $P_{1}$ and $P_{2}$, there is a unique fold placing $P_{1}$ onto $P_{2}$.

Axiom 3: Given two lines $L_{1}$ and $L_{2}$, there is a fold placing $L_{1}$ onto $L_{2}$.

Axiom 4: Given a point $P$ and a line $L$, there is a unique fold perpendicular to $L$ passing through $P$. Axiom 5: Given two points $P_{1}$ and $P_{2}$ and a line $L$, there is a fold placing $P_{1}$ onto $L$ and passing through $P_{2}$.

Axiom 6: Given two points $P_{1}$ and $P_{2}$ and two lines $L_{1}$ and $L_{2}$, there is a fold placing $P_{1}$ onto $L_{1}$ and $P_{2}$ onto $L_{2}$.

Axiom 7: Given a point $P$ and two lines $L_{1}$ and $L_{2}$, there is a fold placing $P$ onto $L_{1}$ and perpendicular to $L_{2}$.

For a slightly different approach to these axioms and for the connection to Galois theory, we refer to the book by Cox [6].

Origami constructions, contrary to rule-andcompass ones, allow one to trisect any angle (see Figure 7), to double a cube, or to construct a regular heptagon.

Another topic, more closely related to what we discuss in this paper, is the problem of finding an algorithm to decide whether a given crease pattern can actually be folded to generate a physical origami, i.e., a rigid map without interpenetration. The problem turns out to be NP-hard (nondeterministic polynomial-time hard), see [4].

Other interesting research is devoted to constructing crease patterns to fold to some given three-dimensional models with elaborated shape. With suitable software, Lang [21] has been able to fold realistic models of different kinds of animals.
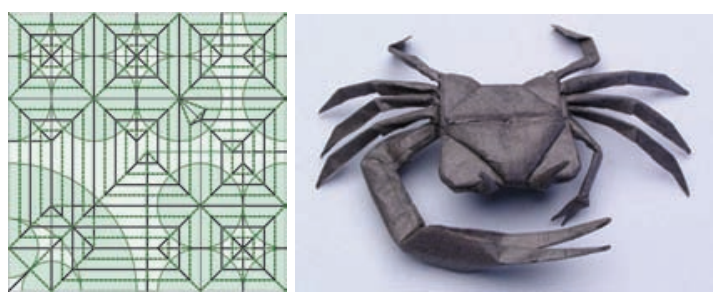

Figure 8. On the left-hand side the "ball packing technique" to create the crease pattern of the model (crab) shown on the right (by R. T. Lang).
One of the main points turns out to be that of finding the best possible packing of given disks into the paper rectangle (see the left-hand side in Figure 8); in fact, we notice that every origami is a short map, namely

$$
|u(x)-u(y)| \leq|x-y|, \quad \forall x, y \in \Omega ;
$$

this means that the distance of two points in the folded model cannot be larger than the distance of the two points in the unfolded model. This implies, for example, that the preimage of the claws of the crab in Figure 8 must contain the whole circle centered in the preimage of the tip of the claw with radius given by the length of the claw.

Recently in [19] some numerical algorithms have been found to reproduce three-dimensional curved models. Some of them have the peculiar property that the crease pattern is mostly composed of curved lines, whereas classical origami only uses straight lines. Curved creases have also been considered by Huffman [15].

Another interesting application comes from aerospace engineering. A team of scientists at the Lawrence Livermore National Laboratory in the United States are building a large space telescope. To put it into action in space, the telescope will be folded and unfolded following the rules of origami. The picture in Figure 9, taken from [14], represents a prototype of the telescope.
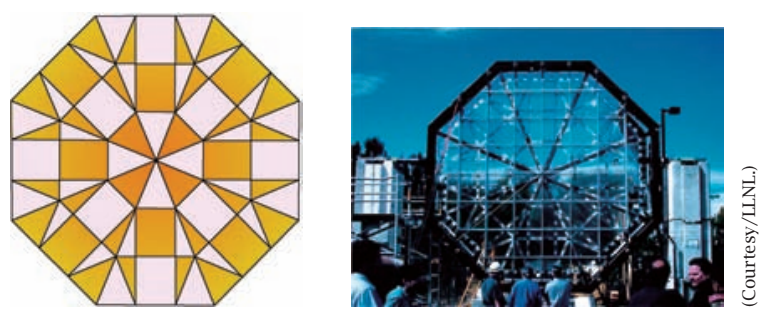

Figure 9. The unfolded space telescope and, on the left, the corresponding singular set.

\section{Mathematical Origami as a Rigid Map}

In this presentation an origami will be modeled by the notion of rigid map. A map $u: \Omega \subset \mathbb{R}^{n} \rightarrow \mathbb{R}^{m}$ is said to be a rigid map if it is Lipschitz continuous and $D u(x) \in O(m, n)$ for a.e. $x \in \Omega$. 
From the definition it follows that, given any two vectors $\sigma, \tau \in \mathbb{R}^{n}$, we have

$$
\begin{aligned}
\langle D u(x) \sigma, D u(x) \tau\rangle_{\mathbb{R}^{m}} & =\left\langle(D u(x))^{t} D u(x) \sigma, \tau\right\rangle_{\mathbb{R}^{n}} \\
& =\langle\sigma, \tau\rangle_{\mathbb{R}^{n}},
\end{aligned}
$$

where $\langle\cdot, \cdot\rangle_{\mathbb{R}^{n}}$ is the scalar product in $\mathbb{R}^{n}$. In particular $u$ leaves invariant the metric on the tangent space.

Since $u$ is Lipschitz continuous it is, by the classical Rademacher theorem, differentiable almost everywhere in $\Omega$. We call the singular set of $u$ the set $\Sigma_{u}$ where the map $u$ is not differentiable. We now quote two classical results. The first one says that isometries are rigid maps with empty singular sets.

Theorem 3.1 (Cartan-Dieudonné). Let $\Omega \subset \mathbb{R}^{n}$ be an open connected set and $u: \Omega \rightarrow \mathbb{R}^{m}$ be an isometry:

$$
|u(x)-u(y)|=|x-y|, \quad \forall x, y \in \Omega .
$$

Then $m \geq n$ and $u$ is affine.

The second result applies to the case $m=n$. In this case a rigid map corresponds to a flat origami. The theorem states that a flat and smooth origami can only be trivial.

Theorem 3.2 (Liouville). Let $\Omega$ be a connected open subset of $\mathbb{R}^{n}$. If $u: \Omega \rightarrow \mathbb{R}^{n}$ is of class $C^{1}$ and satisfies $D u \in O(n)$, then $u$ is affine.

From now on we will only consider piecewise $C^{1}$ rigid maps, which are maps $u: \Omega \rightarrow \mathbb{R}^{n}$ satisfying the following properties: (i) $\Sigma_{u}$ is relatively closed in $\Omega$; (ii) $u$ is of class $C^{1}$ in every connected component of $\Omega \backslash \Sigma_{u}$; (iii) every compact set $K \subset \Omega$ intersects only a finite number of connected components of $\Omega \backslash \Sigma_{u}$.

\section{Reconstruction from the Crease Pattern}

As above, when $n=m$ we are in the flat case, and we consider flat origami, i.e., piecewise $C^{1}$ rigid maps $u: \Omega \subset \mathbb{R}^{n} \rightarrow \mathbb{R}^{n}$. In this context we can prove that, if $\Omega$ is a convex polyhedral set, then every connected component of $\Omega \backslash \Sigma_{u}$ is also a convex polyhedral set. The singular set $\Sigma_{u}$ is the union of the faces bounding the convex regions, hence it is itself a $(n-1)$-dimensional polyhedral complex.

Every $(n-1)$-dimensional facet of $\Sigma_{u}$ is the interface between two different regions, say $A_{1}$ and $A_{2}$. On each region the map $u$ is affine, $u(x)=M_{i} x+q_{i}$ for $x \in A_{i}$, and it is not difficult to show that the two affine maps are related to each other by

$$
M_{2}=M_{1} S, \quad M_{1}=M_{2} S,
$$

where $S \in \mathbb{R}^{n \times n}$ is the matrix representing a symmetry with respect to the $(n-1)$-dimensional plane containing the facet we are considering (thus $\operatorname{det} S=-1$ ). In particular we have

$$
\operatorname{det} M_{2}=-\operatorname{det} M_{1} \text {, }
$$

and the sign of the determinant gives a coloration to $\Omega \backslash \Sigma_{u}$.

In particular we see that if $M_{1}$ is given, $M_{2}$ is uniquely determined. From these considerations it follows that, if $\Omega$ is also connected, once we know the affine map $u$ in a single region, we can recover it in every other region.

However, not every polyhedral complex $\Sigma$ is the singular set of some rigid map $u$. Notice, in particular, that if $e$ is a $(n-2)$-dimensional edge of $\Sigma$, then the number of $(n-1)$-dimensional facets containing $e$ must be even. This is due to the 2 -coloration property of the complement of $\Sigma_{u}$. So we denote with $f_{1}, \cdots, f_{2 k}$ the faces containing $e$. If we denote with $\alpha_{i}$ the angles between faces $f_{i}$ and $f_{i+1}$, by means of condition (2) we can show that the following angle condition holds (see Figure 10):

$$
\sum_{i=1}^{k} \alpha_{2 i-1}=\sum_{i=1}^{k} \alpha_{2 i}=\pi .
$$

This condition is well known in the study of flat origami. When $n=2$ this condition was first observed by Kawasaki [18].

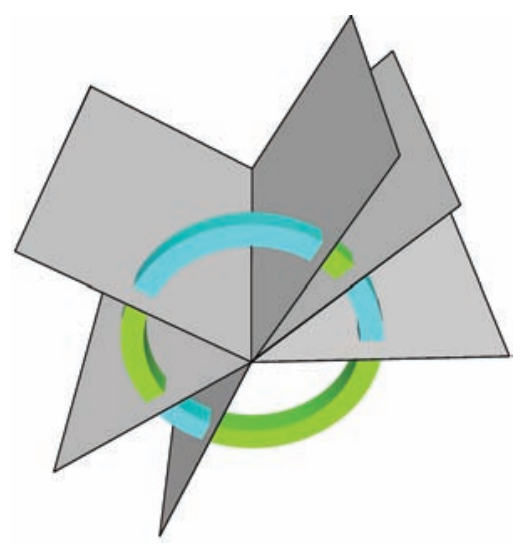

Figure 10. The angle condition. The sum of angles with the same color is equal to $\pi$.

Hence, up to now, we know that the singular set $\Sigma_{u}$ of a piecewise $C^{1}$ rigid map $u$ is a polyhedral set such that $\Omega \backslash \Sigma_{u}$ is 2-colorable; moreover, every $(n-2)$-dimensional edge is contained in an even number of $(n-1)$-dimensional facets spaced so that the sum of angles on the regions of one color is equal to the sum of angles for the regions of the other color.

It is interesting to know that the reverse implication, stated in the following theorem, holds (see [10]).

Theorem 4.1 (Recovery theorem). Let $\Omega$ be a simply connected open subset of $\mathbb{R}^{n}$. Let $\Sigma \subset \Omega$ be a locally finite polyhedral set satisfying the angle condition (3) on every $(n-2)$-dimensional facet. Then

(i) there exists a rigid map $u$ such that $\Sigma=\Sigma_{u}$ is the singular set of $\mathrm{Du}$; 
(ii) $u$ is uniquely determined once we fix the value $y_{0}=u\left(x_{0}\right)$ and the Jacobian $J_{0}=D u\left(x_{0}\right)$ at a point $x_{0} \in \Omega \backslash \Sigma$.

The theorem means that if we construct a set $\Sigma$ satisfying the conditions just mentioned, there always exists a map $u$ such that $\Sigma=\Sigma_{u}$. Moreover, the map $u$ is uniquely determined up to compositions (on the left) with rigid motions of the whole space.

In the literature about flat folding (see, for instance, [2], [4]), the problem of reconstructing an origami from its singular set (which is also called crease pattern) has already been considered. However, a more strict definition of origami should be used to treat physical origami. In a physical origami the interpenetration problem is addressed, i.e., the problem that physically a noninjective map cannot be folded without cutting the paper. To be more precise, since the thickness of the paper can anyway be neglected, we admit noninjective maps as long as they are the limit of injective ones.

This restriction makes the angle condition not sufficient to guarantee the global reconstruction of a physical origami. An example is given by the singular set in Figure 11 which satisfies the angle condition, hence it is the singular set of some map $u$ which, however, cannot be folded without cutting the paper. In this more strict setting it is shown (see [4]) that the problem of deciding whether a crease pattern can be flat-folded is NP-hard.

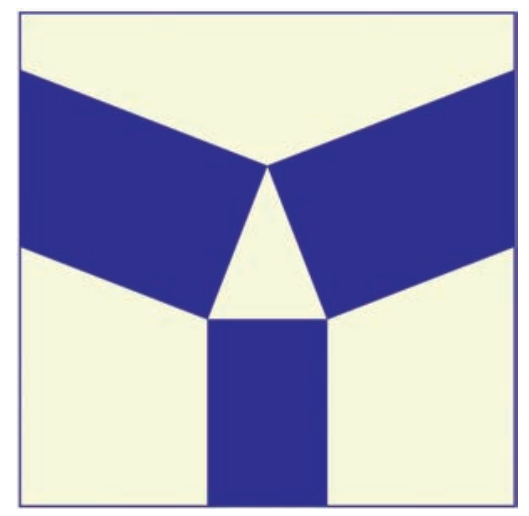

Figure 11. A singular set of a rigid map which cannot actually be folded without cutting the paper.

\section{Fractal Construction}

We now turn our attention to the more difficult problem of finding a solution to the following implicit partial differential system

$$
\left\{\begin{array}{cl}
D u(x) \in O(n) & \text { for a.e. } x \in \Omega \\
u(x)=0 & \text { for } x \in \partial \Omega
\end{array}\right.
$$

in the case when $\Omega$ is a rectangle in $\mathbb{R}^{2}$ (or parallelepiped in $\mathbb{R}^{n}$ ). Now we know that a solution is represented by a flat origami. However, the Dirichlet boundary condition is, in principle, incompatible with the first-order differential system, as already mentioned in the introduction.

We first discuss the case $n=2$. As we already said, only a fractal construction can ensure the boundary condition $u=0$. Figure 6 gives an idea of the construction that we are going to develop. If $\Omega$ is a square, we can divide it in infinitely many squares, which are smaller and smaller, while we approach the boundary of $\Omega$. Then it is enough to consider a base map $u_{0}$ defined on one of these squares. This map will be translated, rotated, and rescaled to fit any other square. To assure that the gluing of the squares gives a continuous map, we need the base map to have prescribed recursive boundary conditions. More precisely we require that, on the right-hand side of the base square, the map is defined so that it reproduces twice the values of the left-hand side, rescaled by half; i.e.,

$$
\begin{array}{cc}
u_{0}(1, y)=u_{0}(0,2 y) & \text { for } y \in[0,1 / 2], \\
u_{0}(1, y)=u_{0}(0,2 y-1) & \text { for } y \in[1 / 2,1] ;
\end{array}
$$

while on the upper and lower sides we only need periodic boundary conditions:

$$
u_{0}(x, 0)=u_{0}(x, 1) \quad \text { for } x \in[0,1] .
$$

If the map assumes at least once the value 0 on every square in the net, then by its Lipschitz continuity (every rigid map is 1-Lipschitz continuous) it can be extended to the boundary $\partial \Omega$ with the 0 value.

The base module can thus be found by using the angle condition (3) and the recovery theorem 4.1. In this way we are able to find solutions to the above problem. More generally we might consider linear boundary conditions, such as $u(x, y)=(\lambda x, \mu y)$ on $\partial \Omega$. The corresponding singular set is shown in Figure 12.

Notice that we can easily pose the problem in higher dimension by considering $n$-dimensional flat origami, namely hyper-origami. Again we subdivide the domain in smaller and smaller cubes (or parallelepipeds). On the base domain we need to find an $n$-dimensional origami which fulfills the recursive boundary condition. For $n=3$ we can imagine this operation as the folding of a rigid three-dimensional box. In Figure 13, we present the "instructions" for folding the base parallelepiped to obtain the zero boundary condition. By gluing together the base modules we obtain the 3-dimensional singular set.

Finally we note that one can solve (4) with symmetric rigid maps, i.e., maps $u$ which are gradients of functions $v: \Omega \subset \mathbb{R}^{2} \rightarrow \mathbb{R}$. The problem (4) when $n=2$ then becomes

$$
\left\{\begin{array}{cl}
D^{2} v(x) \in O(2) & \text { for a.e. } x \in \Omega \\
D v(x)=0 & \text { for } x \in \partial \Omega
\end{array}\right.
$$




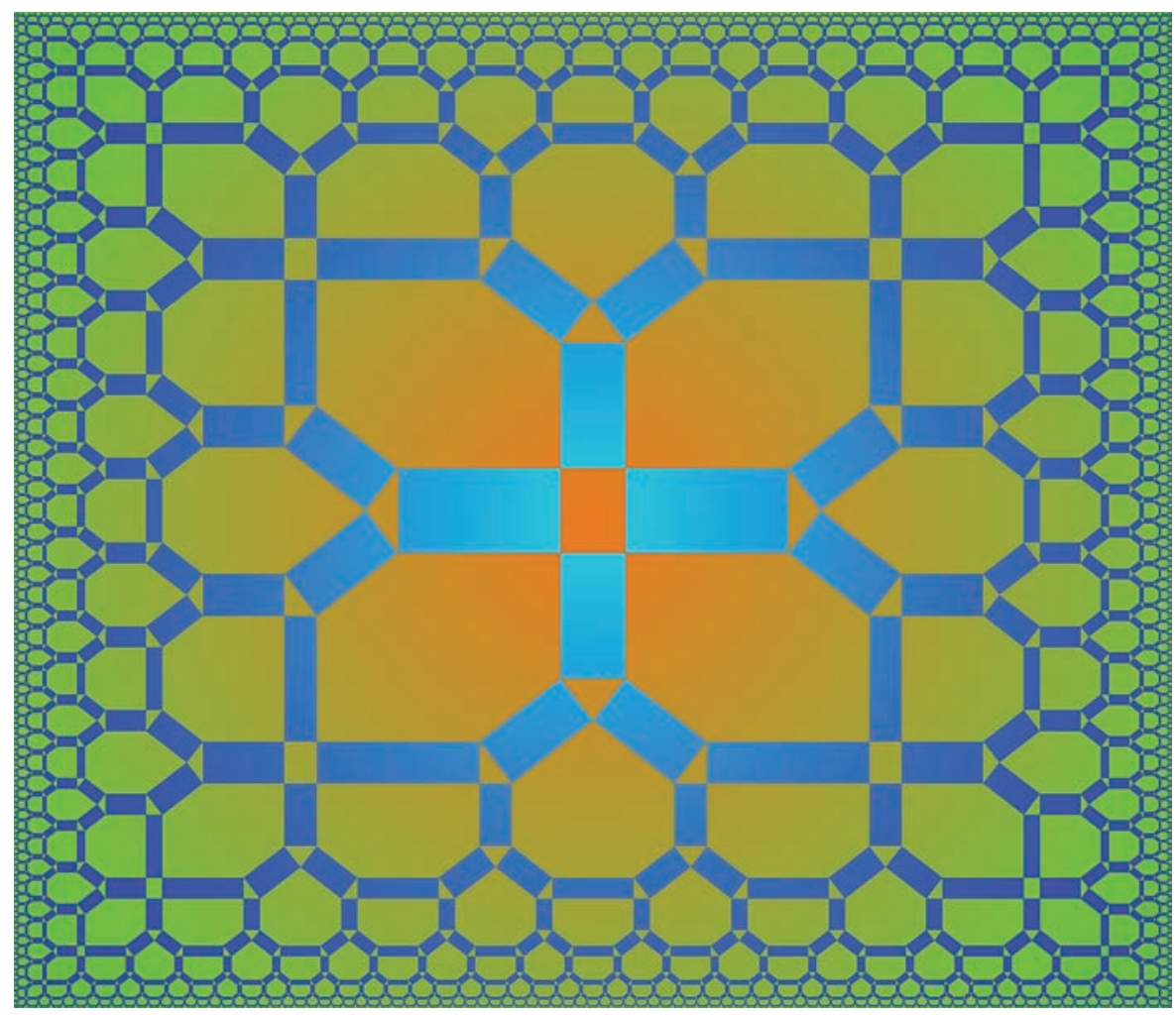

Figure 12. A fractal construction for the linear boundary data.

It turns out (see [11]) that the corresponding angle condition (3) is, in this case, much more restrictive in the sense that at every vertex of $\Sigma_{u}=\Sigma_{D v}$ exactly four consecutive angles $\alpha^{+}, \alpha^{-}, \beta^{+}, \beta^{-}$meet with

$$
\alpha^{+}+\beta^{+}=\pi \quad \text { and } \quad \alpha^{-}=\beta^{-}=\frac{\pi}{2} .
$$

We call this property "second-order angle condition". The singular set represented in Figure 12 satisfies the second-order angle condition, in contrast with the singular set of Figure 6 .

\section{Implicit Partial Differential Equations: Conclusion}

What we have done so far is to find explicit solutions to Dirichlet problems, as in the section "Fractal Construction". This kind of problem belongs to a wider class, called implicit partial differential equations. Namely we look for Lipschitz continuous solutions to

$$
\left\{\begin{array}{cl}
F(x, u(x), D u(x))=0 & \text { for a.e. } x \in \Omega \\
u(x)=\varphi(x) & \text { for } x \in \partial \Omega,
\end{array}\right.
$$

where $u, \varphi: \Omega \subset \mathbb{R}^{n} \rightarrow \mathbb{R}^{m}$ and $F: \Omega \times \mathbb{R}^{m} \times \mathbb{R}^{m \times n} \rightarrow$ $\mathbb{R}$ (also called Hamiltonian).

Note that, as in (4), the problem is apparently ill posed, since we want to solve a first-order PDE and at the same time prescribe full Dirichlet condition on the boundary. Indeed, except for very special cases, no $C^{1}$ solution is to be expected, and only
Lipschitz solution may exist. Curiously enough, if there is a solution of (4) or more generally of (5), then, in general, there are infinitely many solutions. Let us review some of the techniques for solving such equations and see how the constructions we have proposed for origami may shed light on some issues related to implicit PDEs.

Let us start with the scalar case $m=1$ which has received considerable attention since the work of E. Hopf, notably by Kruzkov, Lax, and Oleinik. In this case the equation

$$
F(x, u, D u)=0
$$

is sometimes called Hamilton-Jacobi equation. Crandall-Lions further developed in this context the important tool of viscosity solution. The beauty and the importance of the viscosity method is that, at the same time, it gives existence of solutions and a way of selecting one among the infinitely many solutions of (5). It is, however, of crucial importance in this context that the Hamiltonian $F$ be convex in the variable $D u$. For example, the eikonal equation

$$
\left\{\begin{array}{cl}
|D u(x)|=1 & \text { for a.e. } x \in \Omega \\
u(x)=0 & \text { for } x \in \partial \Omega
\end{array}\right.
$$

has, for convex domains $\Omega$, the viscosity solution

$$
u(x)=\operatorname{dist}(x, \partial \Omega),
$$




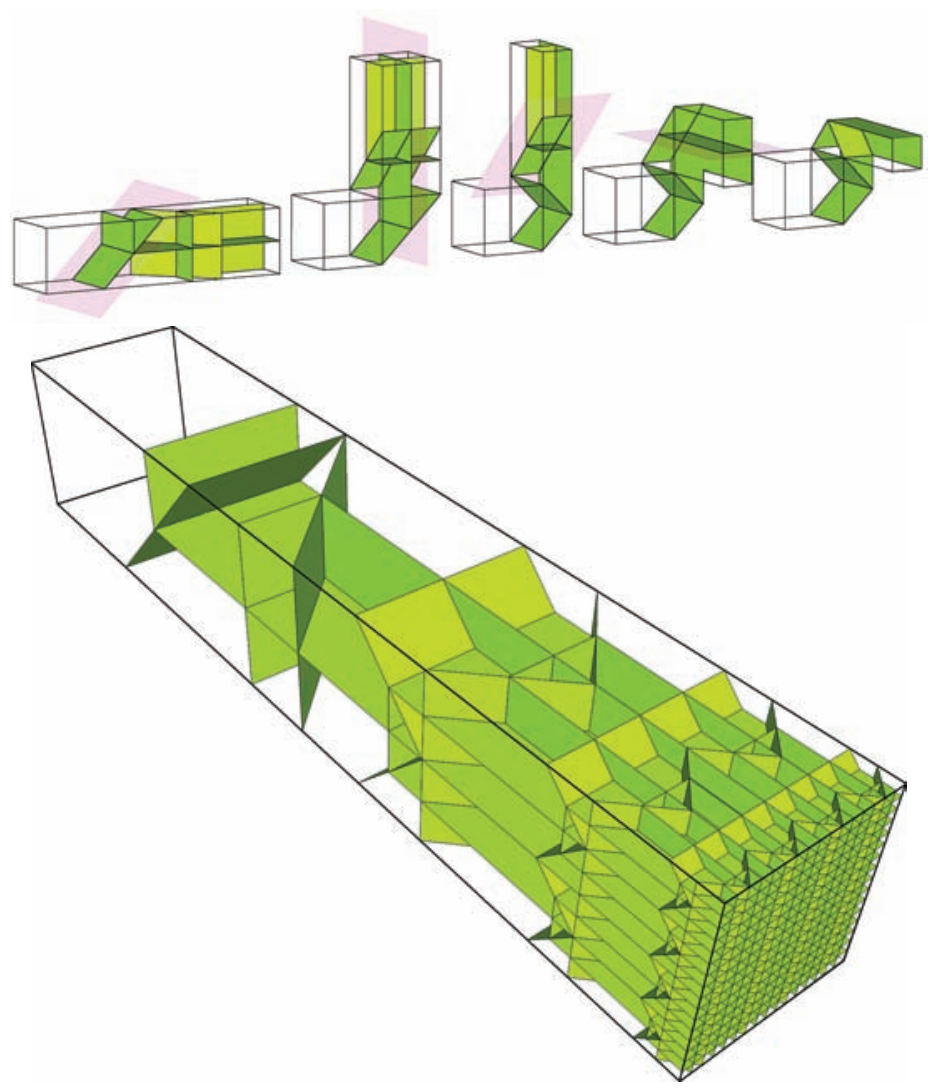

Figure 13. A "hyper-origami", precisely a three-dimensional flat origami which solves the Dirichlet problem (4) for $n=3$ with zero boundary condition. At the top an elementary brick is represented. It has a self-similar property which allows recursively gluing it together with half-sized homothetic copies of itself to obtain the pattern represented at the bottom.

although, as said earlier, there are infinitely many other solutions, one of them being

$$
u(x)=-\operatorname{dist}(x, \partial \Omega) .
$$

However, as soon as the Hamiltonian $F$ is nonconvex (with respect to the last variable) and still in the scalar case $m=1$, or in the vectorial context $n, m \geq 2$ (as for origami), the viscosity theory does not apply. Two other main theories have been developed to deal with these cases, and, although different, they give essentially the same results (for detailed references see [9]). However, both theories are purely existential and do not give a simple criterion for selecting one solution among the infinitely many as did the viscosity theory. Let us briefly discuss both of them.

(i) The theory of convex integration proposed by Gromov is one of them. It was originally introduced to give a new proof of the celebrated Nash-Kuiper theorem on isometric embedding, which is closely related to the problem discussed in the present paper. The theory of convex integration has been developed in the context of implicit partial differential equations by Müller-Sverak and others. (ii) The Baire category method is the other tool and has been introduced by Cellina, Bressan-Flores, and De Blasi-Pianigiani for the scalar case and generalized to the vectorial context by DacorognaMarcellini.

We also point out that, in the scalar case $m=1$, and for Hamiltonians $F$, not necessarily convex, that do not depend on lower-order terms (i.e., $F(D u)=0$ ), explicit solutions can easily be obtained and are known as "pyramids", following the work of Cellina and Friesecke. This was a source of inspiration to construct explicit solutions of (4) to Cellina-Perrotta and to the work presented here. Contrary to the finite element method used in numerical analysis to build approximated solutions, here, by means of piecewise affine maps with appropriate grid, we are able to exhibit exact solutions.

Since the two approaches described above are only existential, it is then an important issue to single out one solution among the infinitely many. It is what we achieved by means of origami for the special problem (4). There are many other problems related, for example, to geometry, optimal design, 
or nonlinear elasticity where an actual explicit construction would be important.

Among them a problem which is significant in applications to microstructures in crystallographic models is the one of potential wells (see, for example, [3]). When we consider the case of two wells, it consists in finding maps $u$ whose gradient satisfies

$$
\left\{\begin{array}{cl}
D u(x) \in S O(n) A \cup S O(n) B, & \text { a.e. in } \Omega \\
u(x)=\varphi(x), & \text { on } \partial \Omega,
\end{array}\right.
$$

where $A$ and $B$ are two given $n \times n$ matrices and $S O(n)$ is the set of special orthogonal matrices; i.e., those with determinant equal to +1 . This is a natural generalization of the problem considered here (see (4)), since

$$
O(n)=S O(n) I \cup S O(n) I_{-},
$$

where $I$ is the identity matrix and $I_{-}=$ $\operatorname{diag}(-1,1, \ldots, 1)$.

Finally, implicit partial differential equations, because of the existence of infinitely many solutions, are particularly challenging for numerical analysts. In the problem studied in the section "Fractal Construction", one could imagine, as has been suggested by one of the referees, finding algorithms that mimic the origami construction.

\section{References}

[1] R. C. AlPERIN, A mathematical theory of origami constructions and numbers, New York J. Math. 6 (2000), 119-133.

[2] E. M. Arkin, M. A. Bender, M. A. Demaine, et al., When can you fold a map?, Computational Geometry 29 (2004), 23-46.

[3] J. M. BALL and R. D. JAMES, Proposed experimental tests of a theory of fine microstructure and the two wells problem, Phil. Trans. Royal Soc. London A 338 (1991), 389-450.

[4] M. BERN and B. HAYES, The complexity of flat origami, Proceedings of the 7th Annual ACM-SIAM Symposium on Discrete Algorithms, 1996, pp. 175-183.

[5] B. CASSElman, If Euclid had been Japanese, Notices Amer. Math. Soc. 54 (2007), 626-628.

[6] D. A. Cox, Galois Theory, Wiley-Interscience, Hoboken, NJ, 2004.

[7] M. G. Crandall, H. Ishit, and P. L. Lions, User's guide to viscosity solutions of second order partial differential equations, Bull. Amer. Math. Soc. 27 (1992), 1-67.

[8] B. DACOROGNA and P. MARCELLINI, General existence theorems for Hamilton-Jacobi equations in the scalar and vectorial case, Acta Mathematica 178 (1997), $1-37$.

[9] B. DACorogna and P. MARCELLINI, Implicit Partial Differential Equations, Progress in Nonlinear Differential Equations and Their Applications, vol. 37, Birkhäuser, 1999.

[10] B. DAcorogna, P. MARcellini, and E. PAOlini, Lipschitz-continuous local isometric immersions: Rigid maps and origami, J. Math. Pures Appl. 90 (2008), 66-81.
[11] B. Dacorogna, P. Marcellini, and E. PAolini, Functions with orthogonal Hessian, Differential and Integral Equations 23 (2010), 51-60.

[12] M. Gromov, Partial Differential Relations, SpringerVerlag, Berlin, 1986.

[13] K. HAgA, Origamics, Mathematical Explorations Through Paper Folding, World Scientific, Singapore, 2009.

[14] A. Heller, A giant leap for space telescopes, Science \& Technology Review, March (2003), 12-18.

[15] D. Huffman, Curvature and creases: A primer on paper, IEEE Trans. on Computers C-25 (1976), 10101019.

[16] T. Hull, On the mathematics of flat origamis, Congressus Numerantium 100 (1994), 215-224.

[17] T. Hull, Project origami. Activities for exploring mathematics, A K Peters, Ltd., Wellesley, MA, 2006.

[18] T. KAWASAKI, On the relation between mountain creases and valley creases of a flat origami, Proceedings of the 1st International Meeting of Origami Science and Technology, Ferrara (H. Huzita, ed.), 1989, pp. 229-237.

[19] M. Kilian, S. Floery, Z. Chen, N. Mitra, A. ShefFer, and H. PotTMAnN, Curve Folding, ACM SIGGRAPH, 2008.

[20] N. H. KUIPER, On $C^{1}$-isometric imbeddings I, Nederl. Akad. Wetensch. Proc. Ser. A. 58 (1955), 545-556.

[21] R. J. LANG, A computational algorithm for origami design, Proceedings of the 12th Annual ACM Symposium on Computational Geometry, 1996, pp. 98-105.

[22] J. NASH, $C^{1}$-isometric imbeddings, Annals of Mathematics 60 (1954), 383-396. 